Document downloaded from:

http://hdl.handle.net/10251/78766

This paper must be cited as:

Patrao Herrero, I.; González Medina, R.; Marzal-Romeu, S.; Garcerá Sanfeliú, G.; Figueres Amorós, E. (2017). Synchronization of power inverters in islanded microgrids using an FMmodulated signal. IEEE Transactions on Smart Grid. 8(1):503-510. doi:10.1109/TSG.2016.2574038.

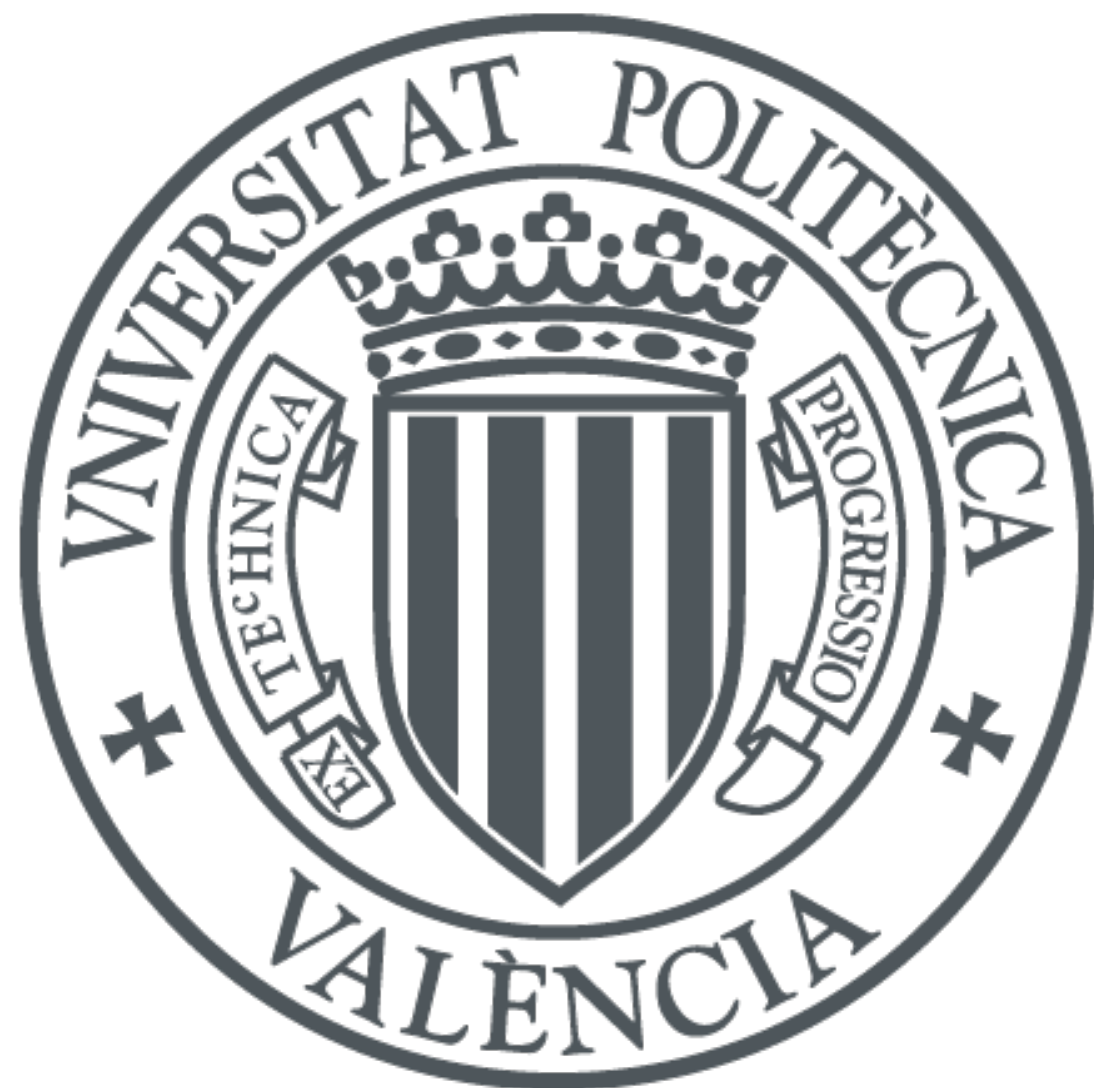

The final publication is available at

http://dx.doi. org/10.1109/TSG.2016.2574038

Copyright Institute of Electrical and Electronics Engineers (IEEE)

Additional Information

"(c) 2017 IEEE. Personal use of this material is permitted. Permission from IEEE must be obtained for all other users, including reprinting/ republishing this material for advertising or promotional purposes, creating new collective works for resale or redistribution to servers or lists, or reuse of any copyrighted components of this work in other works." 


\title{
Synchronization of power inverters in islanded microgrids using an FM-modulated signal
}

\author{
I. Patrao, R. González-Medina, S. Marzal, G. Garcerá, Member, IEEE and E. Figueres, \\ Senior Member, IEEE
}

\begin{abstract}
A microgrid can operate in island mode, isolated from the main grid during certain time intervals. When operated in island mode, the electronic converters of the microgrid must keep the voltage and frequency of the microgrid inside the desired range. The converters of a microgrid can be classified into three groups: grid-feeding, grid-supporting and gridforming power converters. The grid-forming converters operate as a voltage source, and require an external synchronization signal provided by the Microgrid Central Controller (MCC).

Both the noise and the delay in the synchronization signals received by the grid-forming converters are critical issues, which deteriorate the quality of the microgrid voltage and may overload those converters. The synchronization signals must be robust and suitable for operating in noisy environments.

In this paper, the synchronization signal is frequencymodulated (FM) to be transmitted, being robust against noise. The transmission is done through an industrial RS-485 line with a low delay. The demodulation is performed with a low computational load by the control processors of the grid-forming power converters.
\end{abstract}

Index Terms - Microgrids, Grid-forming converters, Synchronization, Voltage-source, Islanded operation

\section{INTRODUCTION}

$\mathrm{N}$ ew trends in the distribution of electric energy point to microgrids [1] as a scenario in which a part of the electrical grid can be disconnected from the main grid during certain time intervals for security, quality of service or economic issues [2]. In this context the distributed generators and the power electronic interfaces operating in the microgrid are critical for the reliability of the system. A typical AC microgrid is a cluster formed by three different type of devices: distributed generators, energy storage systems (batteries, flywheels, ultracapacitors, etc) and loads.

The Institute of Electrical and Electronics Engineers (IEEE) provides the following definition for the distributed generators: "The generation of electricity by facilities sufficiently smaller than central generating plants as to allow interconnection at nearly any point in a power system" [3]. So that, the maximum power of a distributed generator is limited to a few megawatts (10-50 MW) [3, 4]. However, most distributed generators feature a maximum power below $200 \mathrm{~kW}$ and operate by means of power electronic converters

This work has been supported by the Spanish Ministry of Economy and Competitiveness (MINECO) under grant ENE2015-64087-C2-2-R, which was partially cofinanced by the European Regional Development Fund (ERDF) .

I. Patrao, R. González-Medina, S. Marzal, E. Figueres and G. Garcerá are with Grupo de Sistemas Electrónicos Industriales del Departamento de Ingeniería Electrónica, Universitat Politècnica de València, Camino de Vera s/n, 46022 Valencia, Spain. (e-mail: ivpather@upv.es)
$[5,6]$

The power converters used in the distributed generators of an AC microgrid can be classified into three groups, depending on their operation principle: grid-feeding, gridsupporting and grid-forming power converters [7].

A grid-feeding power converter behaves as a current source that injects power into an 'established' microgrid. These converters are not capable of setting the microgrid voltage and frequency on their own; they just provide power to the energized loads. The current injection must be accurately synchronized with the grid voltage to avoid the injection of reactive power, usually by means of a phase-locked loop (PLL) algorithm [8].

Grid-supporting power converters behave as synchronous generators, adjusting their output frequency and voltage as a function of the active and reactive power delivered. The usual control scheme is commonly known as 'droop control' [9-15], which makes possible to operate a grid-supporting power converter in combination with a grid-forming converter or with other grid-supporting converters. The droop control method does not strictly require any kind of communication signals among converters, but it's usual to include some communications to improve both voltage and frequency regulation and the power sharing among the distributed generators on stream $[11,16]$.

Grid-forming power converters nearly behave like an ideal $\mathrm{AC}$ voltage source, setting the amplitude and frequency of the microgrid voltage. As voltage sources, these converters have a low output impedance, requiring their parallel operation an accurate synchronization among them [7]. By programming a virtual output impedance of the grid-forming power converters it is possible to manage the power sharing between them, so that grid-forming converters of different nominal power levels can work together to set the voltage and frequency of a microgrid.

Grid-forming power converters can be synchronized by means of an external synchronization signal generated by a microgrid central controller (MCC) $[11,17]$, which allows the parallel operation of a great number of this kind of converters (figure 1). Moreover, the MCC can manage the reconnection of the islanded microgrid with the electrical grid, adjusting the phase and frequency of the microgrid to agree with those of the grid before reconnecting. The accurate synchronization of the microgrid phase and frequency before reconnection avoids large transients of active and reactive power after reconnection [18].

It is difficult to start an islanded microgrid composed by several low power generators if the loads draw more power 
than that available in a single converter. This is also known as 'black start', which is an issue under discussion in distributed generation [19]. Using grid-forming converters operating in parallel by means of an external synchronization signal provides a behavior similar to that of a single AC voltage source whose nominal power is the sum of those of the converters on stream, enabling a smooth start of the microgrid.

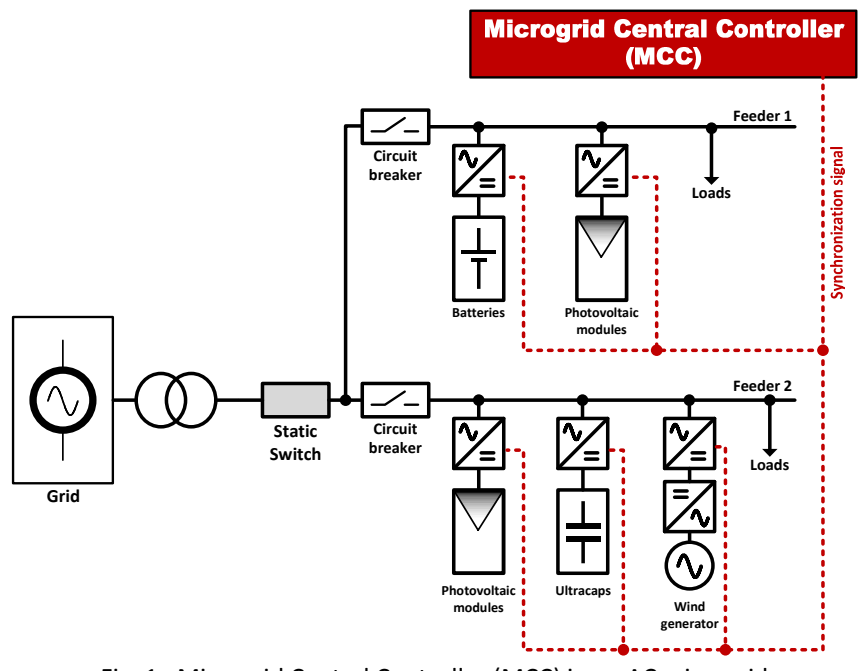

Fig. 1. Microgrid Central Controller (MCC) in an AC microgrid

In this paper it is proposed a frequency-modulation (FM) technique for sending a grid-frequency synchronization signal through a copper twisted-pair from the MCC to the distributed generators operating as grid-forming converters. The FM modulation provides a high robustness against noise and voltage drop in the transmission wire.

The synchronization signal is used by the grid-forming converters as frequency and phase reference for generating the output voltage. With all the converters perfectly synchronized, the use of an output virtual impedance permits to connect them in parallel.

In this paper two grid-forming converters operating in parallel in an islanded microgrid have been tested. They feed an islanded load regulating the output voltage, i.e. the microgrid voltage. The purpose of this paper is to present a synchronization technique among grid-forming inverters. The used load-sharing technique is a simple one which offers acceptable results.

\section{GENERATION AND TRANSMISSION OF THE SYNCHRONIZATION SIGNAL}

\section{A. FM MODULATION}

To avoid the influence of the voltage drop in the transmission wires or noise problems, the synchronization signal is modulated in frequency (FM). The proposed modulated signal is a square waveform, easy to generate by means of the PWM unit of a microcontroller, i.e. using a fixed $50 \%$ duty cycle and changing the period dynamically. The frequency adopted for the carrier signal is $F_{C}=10 \mathrm{kHz}$, much higher than the frequency of the modulated signal (50 or 60
$\mathrm{Hz}$ ), and the frequency sweep is $\pm 100 \mathrm{~Hz}(\Delta \mathrm{f}=100 \mathrm{~Hz})$, so that the frequency of the square signal is in the range $9.9 \mathrm{kHz}-$ $10.1 \mathrm{kHz}$. A microgrid frequency of $50 \mathrm{~Hz}$ has been chosen for the analysis. Thus, the square signal sweeps from $9.9 \mathrm{kHz}$ to $10.1 \mathrm{kHz}$ with a periodicity of $20 \mathrm{~ms}(50 \mathrm{~Hz})$.

The sweep range of $\pm 100 \mathrm{~Hz}$ has been chosen to avoid digital resolution problems in the controller of the gridforming converters during the demodulation process. If an application requires a narrow frequency sweep, this could be achieved with a carefully design of the frequency measurement for the specific microcontroller used in that application. The aim of this paper is to provide a methodology that can be used in a wide range of applications.

The modulation process is shown in figure 2. The signal with the frequency $\left(\mathrm{f}_{\mathrm{GRID}}\right)$ and phase information of the grid voltage is modulated in frequency giving rise to a square signal with a variable frequency which is sent through a transmission medium, e.g. a CAT5e twisted pair, with a propagation delay lower than $1 \mu \mathrm{s} / 100 \mathrm{~m}[20]$.

In the modulation stage the synchronization signal $(\mathrm{x}=$ $\cos \left(2 \cdot \pi \cdot f_{G R I D} \cdot t\right)$, in the range \pm 1 , is transformed into a frequency sweep around the carrier signal frequency (Frec $=$ $\mathrm{F}_{\mathrm{C}}+\Delta \mathrm{f} \cdot \mathrm{x}$ ). In the next step the frequency information (Period $\left.=1 / F_{C}\right)$ is loaded into the PWM unit of a microcontroller to obtain a $50 \%$-duty square signal of variable frequency. This digital signal, TTL/CMOS output of the PWM hardware unit, is introduced into a transceiver (e.g. an RS-485 transceiver) and transmitted through the physical medium.

Neither the voltage noise nor the voltage drop in the transmission wires will affect the information, since it's coded in the frequency instead of in the voltage amplitude.

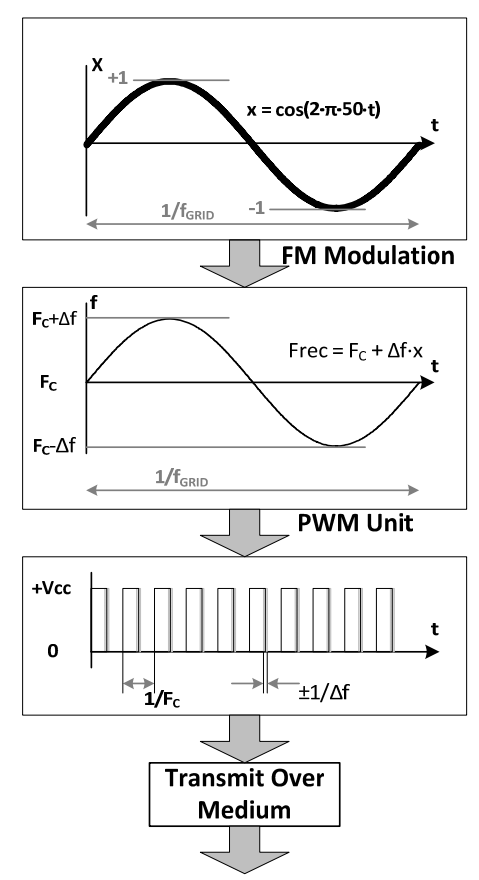

Fig. 2. Modulation stage

\section{B. DEMODULATION}

The digital controller of any grid-forming converter in the 
microgrid has to be capable of demodulating the FM signal with a low processing overhead. In this case a Texas Instruments TMS320F28335 Delfino microcontroller is responsible for capturing the square signal and calculating its period using the compare-and-capture hardware unit [21], thus being a very fast and reliable process.

The inverse of the period, read by the capture-and-compare unit, is the instantaneous frequency of the modulated signal. The frequency information is introduced into the demodulator stage, which rebuilds the original signal for the power converter synchronization (Figure 3 ).

The demodulator stage uses a phase-locked loop (PLL), shown in figure 3 , to rebuild the synchronization signal from the frequency signal generated by the compare-and-capture unit. The output signal of the demodulator stage is in the range \pm 1 , so it can be used directly to generate a sinusoidal output voltage reference, avoiding the use of trigonometric operations in the digital signal controller.

All the grid-forming converters are internally programmed with the same RMS value for the grid voltage reference. The grid voltage reference is multiplied by the output signal of the demodulator to obtain the instantaneous output voltage reference of each grid-forming converter on stream.

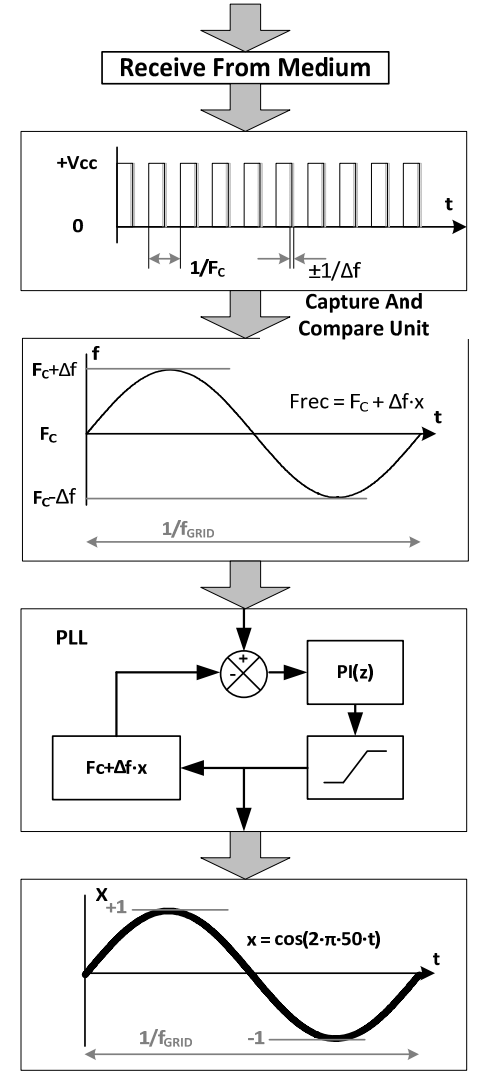

Fig. 3. Demodulation stage.

\section{THE DELAY ISSUE}

The synchronization signal can be received with a small time delay between the converters because of the different physical location of the grid-forming converters in the microgrid. The different delays in the synchronization signals received by the generation units produces a small delay between the current injected by each generator, so that reactive power is generated or absorbed by the generators, even with pure-resistive loads.

Figure 4 shows a pair of grid-forming converters (with output voltages $\mathrm{V}_{1}$ and $\mathrm{V}_{2}$ ) connected in parallel forming a low voltage microgrid that feeds a resistive load $\left(\mathrm{R}_{\mathrm{LOAD}}\right)$. The impedance of the electrical lines is assumed resistive, since the low-voltage cabling presents a negligible inductance.

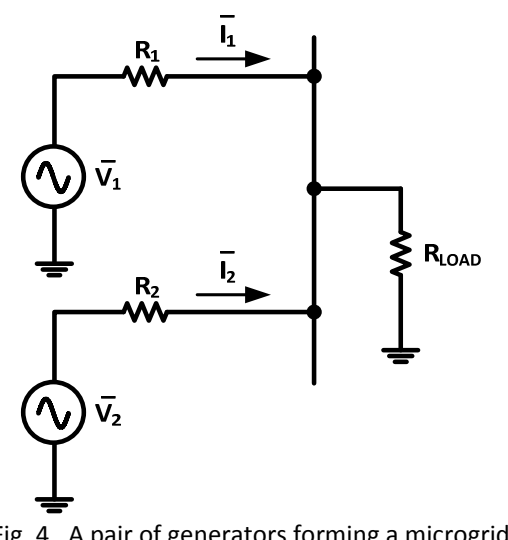

The current phasors for both generators can be calculated as shown in (1-2). Without loss of generality, in the following analysis the phase of $\mathrm{V}_{1}$ has been assumed the phase origin $\left(\overline{V_{1}}=V_{1-R M S \angle 0}\right)$, being $\overline{V_{2}}=V_{2-R M S} \angle \phi_{V 2}$

If the RMS values of $V_{1}$ and $V_{2}$ agree and the phase of the current vectors is small, the phase of the currents $I_{1}$ and $I_{2}$ can be calculated as shown in (3-5). Note that the phase angles of the currents $I_{1}$ and $I_{2}$ drawn from generators $\# 1$ and $\# 2$ are proportional to $\phi_{\mathrm{V} 2}$, but with opposite signs, i.e. the reactive power generated by unit \#1 will be consumed by generator \#2. The voltage phasors are generated from the synchronization signal, so that different delays in the signals received in the generators is a critical issue.

$$
\begin{gathered}
\overline{I_{1}}=\frac{\overline{V_{1}} \cdot\left(1+\frac{R_{2}}{R_{L O A D}}\right)-\overline{V_{2}}}{R_{1}+R_{2}+\frac{R_{1} \cdot R_{2}}{R_{L O A D}}=I_{1} \cdot e^{j \cdot \phi_{I_{1}}}} \\
\overline{I_{2}}=\frac{\overline{V_{2}} \cdot\left(1+\frac{R_{1}}{R_{L O A D}}\right)-\overline{V_{1}}}{R_{1}+R_{2}+\frac{R_{1} \cdot R_{2}}{R_{L O A D}}}=I_{2} \cdot e^{j \cdot \phi_{I_{2}}} \\
\phi_{I_{1}}=-\frac{R_{L O A D}}{R_{2}} \cdot \phi_{V_{2}} \\
\phi_{I_{2}}=\left(1+\frac{R_{L O A D}}{R_{1}}\right) \cdot \phi_{V_{2}}
\end{gathered}
$$

$\Delta \phi_{I}=\phi_{I_{1}}-\phi_{I_{2}}=-\left(1+\frac{R_{L O A D}}{R_{1}}+\frac{R_{L O A D}}{R_{2}}\right) \cdot \phi_{V_{2}}$

The time delay in the synchronization signal received by 
distributed generators placed in different physical locations is mainly due to the propagation delay of the signal over the transmission medium. Considering a maximum length of $1 \mathrm{~km}$ between the most distant distributed generators and using a CAT5e twisted pair wire (with a delay lower than $1 \mu \mathrm{s} / 100 \mathrm{~m}$ ) the delay will be lower than $10 \mu \mathrm{s}$. This delay is equivalent to $\phi_{\mathrm{V} 2}=0.18^{\circ}$ phase difference in the synchronization signal. Even if the ratio $R_{L O A D} / R_{X}$ results in a high value, the phase difference of the currents and the injected reactive power will be kept in low values. This is illustrated in table I for different values of load impedance $\left(\mathrm{R}_{\mathrm{LOAD}}\right)$ and output resistances $\left(\mathrm{R}_{1}\right.$ and $\mathrm{R}_{2}$ ) for a given value of $\phi_{\mathrm{V} 2}$ of $0.18^{\circ}$, corresponding to a long distance $(1 \mathrm{~km})$.

TABLE I: PHASE OFFSET BETWEEN OUTPUT CURRENT $\left(\Phi_{\mathrm{V} 2}=0.18^{\circ}\right)$

\begin{tabular}{|c|c|c|c|}
\hline & $\mathbf{R}_{\mathbf{X}}$ & $\mathbf{R}_{\text {LOAD }}$ & $\Delta \phi_{\mathrm{I}}$ \\
\hline \multirow{2}{*}{$\begin{array}{l}\text { Inverter \#1: 1kVA } \\
\text { Inverter \#2: 1kVA }\end{array}$} & \multirow{2}{*}{$\begin{array}{l}\mathrm{R}_{1}=5 \Omega \\
\mathrm{R}_{2}=5 \Omega\end{array}$} & $\begin{array}{l}52.9 \Omega \\
(1 \mathrm{~kW})\end{array}$ & $-3.9^{\circ}$ \\
\hline & & $\begin{array}{l}26.5 \Omega \\
(2 \mathrm{~kW}) \\
\end{array}$ & $-2.1^{\circ}$ \\
\hline \multirow{2}{*}{$\begin{array}{l}\text { Inverter \#1: } 2 \mathrm{kVA} \\
\text { Inverter \#2: } 1 \mathrm{kVA}\end{array}$} & \multirow{2}{*}{$\begin{array}{c}\mathrm{R}_{1}=2.5 \Omega \\
\mathrm{R}_{2}=5 \Omega\end{array}$} & $\begin{array}{l}52.9 \Omega \\
(1 \mathrm{~kW}) \\
\end{array}$ & $-5.9^{\circ}$ \\
\hline & & $\begin{array}{l}17.6 \Omega \\
(3 \mathrm{~kW}) \\
\end{array}$ & $-2.1^{\circ}$ \\
\hline \multirow{2}{*}{$\begin{array}{l}\text { Inverter \#1: } 5 \mathrm{kVA} \\
\text { Inverter \#2: } 1 \mathrm{kVA}\end{array}$} & \multirow{2}{*}{$\begin{array}{c}\mathrm{R}_{1}=0.5 \Omega \\
\mathrm{R}_{2}=5 \Omega\end{array}$} & $\begin{array}{l}26.45 \Omega \\
(2 \mathrm{~kW}) \\
\end{array}$ & -10.7 \\
\hline & & $\begin{array}{l}10.6 \Omega \\
(5 \mathrm{~kW}) \\
\end{array}$ & $-4.4^{o}$ \\
\hline
\end{tabular}

\section{EXPERIMENTAL RESULTS}

Two inverters have been configured as grid-forming converters to operate in parallel feeding a microgrid. As it can be observed from fig. 1, the MCC sends the synchronization signal to the distributed generators on stream. In the experimental prototype we have used an Agilent 33220A waveform generator as MCC to synthesize the modulated synchronization signal and to send it to the transmission medium. Each one of the inverters has its own TMS320F28335 controller to perform its primary control and the demodulation of the synchronization signal. The same RS485 transceiver (Texas Instruments SN65HVD10) is used in both inverters to convert the synchronization signal from RS485 to a TTL signal. The use of an RS-485 transmission medium and the hardware qualification of the inputs avoids the need of any kind of filtering, reducing to the minimum the time delay in the transmission of the signal.

All the captures have been obtained by means of a Yokogawa DLM4038 8-channel oscilloscope.

The demodulated synchronization signals, with the frequency and phase information, are shown in figure 5, where it is observed that the synchronization signals of both inverters present the same phase and frequency $\left(\mathrm{f}_{\mathrm{GRID}}\right)$. To show the demodulated signals an i2c DAC converter (Texas Instruments DAC7578) has been used for each inverter.

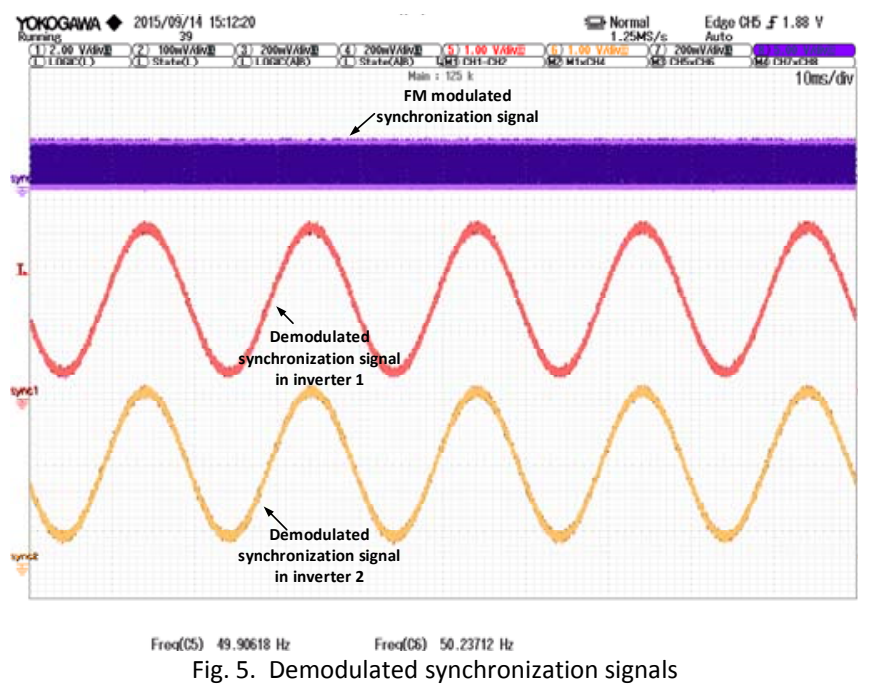

The output virtual impedance, $\mathrm{R}_{\mathrm{V}}$, of each power converter is calculated to produce a $10 \%$ drop of the nominal grid voltage at its maximum power, as shown in equation (5). The EN 50160 norm stablishes a maximum voltage variation in the range $\pm 10 \%$. The no-load output voltage reference of the gridforming converters is set to $230 \mathrm{~V}_{\mathrm{RMS}}+5 \%\left(241.5 \mathrm{~V}_{\mathrm{RMS}}\right)$. Therefore, at full power the output voltage is $230 \mathrm{~V}_{\mathrm{RMS}}-5 \%$ $\left(218.5 \mathrm{~V}_{\mathrm{RMS}}\right)$. Thus, the microgrid voltage varies in the range $230 \mathrm{~V}_{\mathrm{RMS}} \pm 5 \%$, within the limits of the EN 50160. In a first test the inverters are configured as shown in table II, with the same nominal output power ( $1 \mathrm{kVA})$.

$$
R_{V}=\frac{\Delta V_{M A X}}{I_{M A X}}=\frac{\Delta V_{M A X}}{P_{M A X} / V_{G R I D}}
$$

TABLE II: GRID-FORMING CONVERTERS CONNECTED IN PARALLEL

\begin{tabular}{|l|c|c|}
\hline & Inverter 1 & Inverter 2 \\
\hline Nominal Output Voltage & \multicolumn{2}{|c|}{$230 \mathrm{~V} @ 50 \mathrm{~Hz}$} \\
\hline Nominal Output Power & $1 \mathrm{kVA}$ & $1 \mathrm{kVA}$ \\
\hline Output Virtual Impedance & $5 \Omega$ & $5 \Omega$ \\
\hline
\end{tabular}

A $1 \mathrm{~kW}$ highly resistive load is connected to the microgrid formed by both inverters, showing the results of figure 6.a. The microgrid voltage is shown in both channel 1 and channel 3 of the oscilloscope (yellow and pink traces), those channels are used together with channels 2 and 4 for the internal power measurements of the oscilloscope. The total power at the load is $1 \mathrm{kVA}$ (dark blue trace). Inverter \#1 delivers $530.2 \mathrm{VA}$ (53\% of its nominal output power, green trace), whereas Inverter \#2 delivers $503.5 \mathrm{VA}$ (50.4\% of its nominal output power, light blue trace). As expected, the load sharing is done according to the nominal power of the inverters on stream, and each one is operated around $50 \%$ of its nominal power. If the load is incremented to $2 \mathrm{kVA}$ the power is equally shared between the converters, providing each one around the $100 \%$ of its nominal power (figure 6.b). The small displacement power factor that appears in the output currents increases the output current of the inverters, but the output apparent power 
is only slightly higher than the active power, so that the inverters can deliver that extra power without overload.

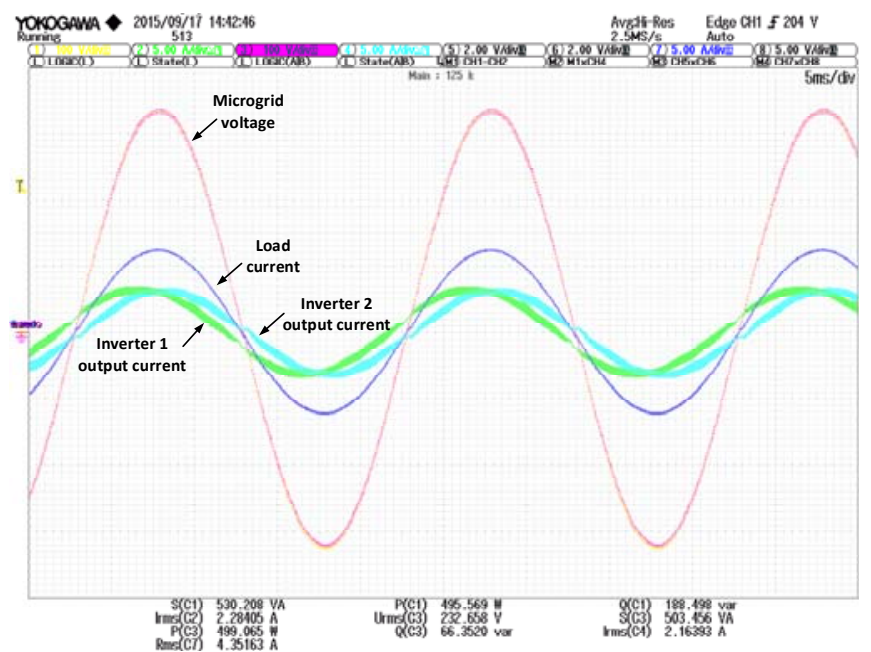

a)

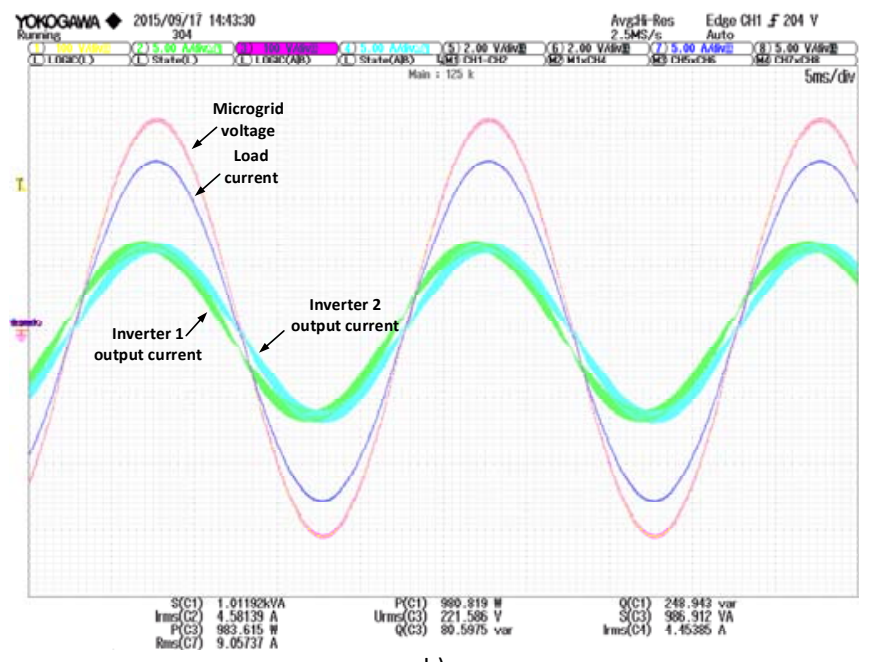

b)

Fig. 6. Load sharing with resistive load and equal nominal power of the inverters. A) Load $=1 \mathrm{~kW}$ b) Load $=2 \mathrm{~kW}$. (Axis scales: 100V/div; 5A/div)

The inverters have been also tested feeding loads that draw reactive power. In the first test, a highly inductive load of 1 $\mathrm{kVAr}$ is powered (figure 7.a). Next, an RL load with a poor power factor $(\mathrm{PF})$ of $1 \mathrm{~kW}+1 \mathrm{kVAr}(1.41 \mathrm{kVA}, \mathrm{PF}=0.71)$ is tested (figure $7 . \mathrm{b}$ ). With the highly inductive load inverter \#1 delivers 426.1 VA (42.6\% of its nominal output power) and inverter \#2 delivers 663.9 VA $(66.4 \%$ of its nominal output power). With the RL load the power delivered by inverter \#1 is $702.8 \mathrm{VA}$ and the power delivered by inverter \#2 is 756 VA. Thus, inverters are loaded at the $70.3 \%$ and $75.6 \%$ of their maximum capacity, respectively.

Besides, a non-linear load with a crest-factor (CF) of 1.6 and a displacement power factor (DPF) of 1 is connected in the microgrid (emulated with a $3.6 \mathrm{~kW}$ programmable electronic load Chroma 63803). Figure 8 shows the waveforms obtained for a load of $2.1 \mathrm{kVA}$. The inverters are equally loaded, sharing the apparent power of the load.
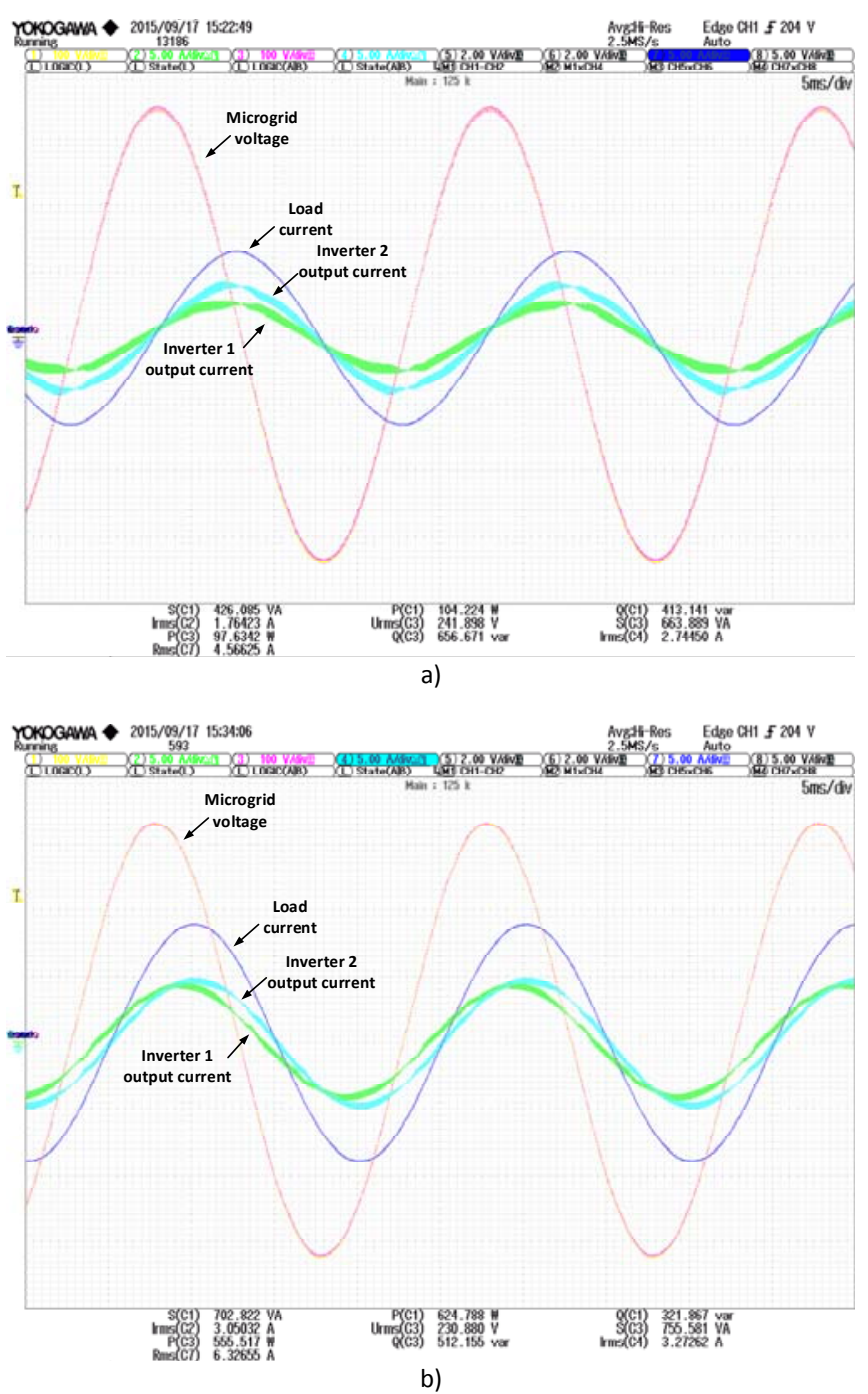

Fig. 7. Load sharing with equal nominal power of the inverters and inductive loads. A) Purely inductive load $1 \mathrm{kVAr}$ b) R-L Load $=1.41 \mathrm{kVA} / \mathrm{PF}=0.71$ (Axis scales: $100 \mathrm{~V} /$ div; $5 \mathrm{~A} /$ div)

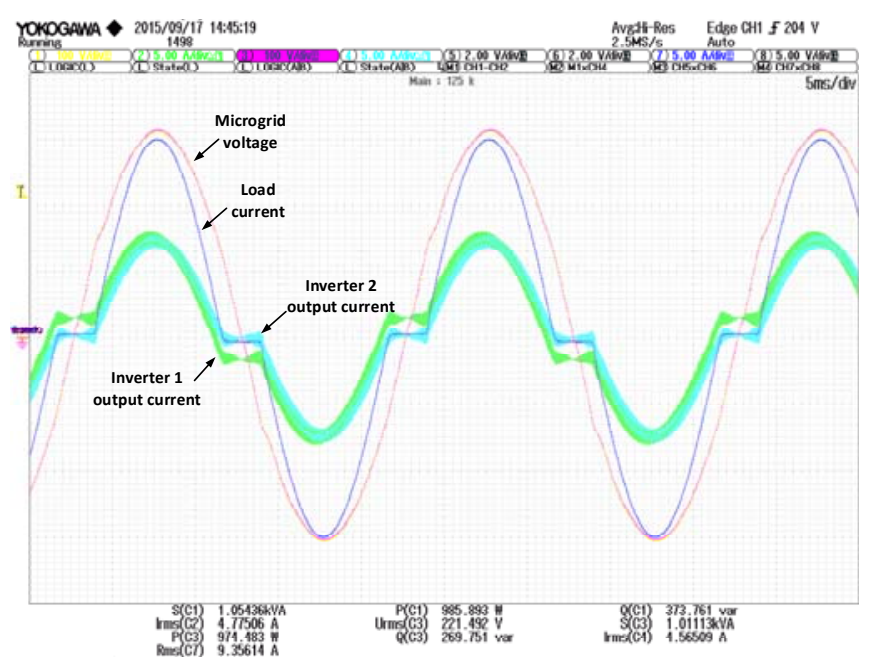

Fig. 8. Load sharing with equal nominal power of the inverters and nonlinear load of 2.4kVA (CF = 1.6, DPF $=1$ ) (Axis scales: $100 \mathrm{~V} / \mathrm{div} ; 5 \mathrm{~A} / \mathrm{div}$ ) 
In the next tests the inverter number 1 is configured for a nominal output power of $2 \mathrm{kVA}$. It is expected that (with the proper adjustment of its virtual output impedance) it delivers more power than the inverter number 2 . The inverters are configured as shown in table III.

TABLE III: GRID-FORMING CONVERTERS CONNECTED IN PARALLEL

\begin{tabular}{|l|c|c|}
\hline & Inverter 1 & Inverter 2 \\
\hline Nominal Output Voltage & \multicolumn{2}{|c|}{$230 \mathrm{~V} @ 50 \mathrm{~Hz}$} \\
\hline Nominal Output Power & $2 \mathrm{kVA}$ & $1 \mathrm{kVA}$ \\
\hline Output Virtual Impedance & $2.5 \Omega$ & $5 \Omega$ \\
\hline
\end{tabular}

In figure 9 a highly resistive load of $3 \mathrm{~kW}$ is connected to the microgrid. The power delivered by the inverter \#1 is 1.97 kVA $(98.5 \%$ of its nominal output power), whereas the power delivered by the inverter \#2 is $0.99 \mathrm{kVA}(99.6 \%$ of its nominal output power).

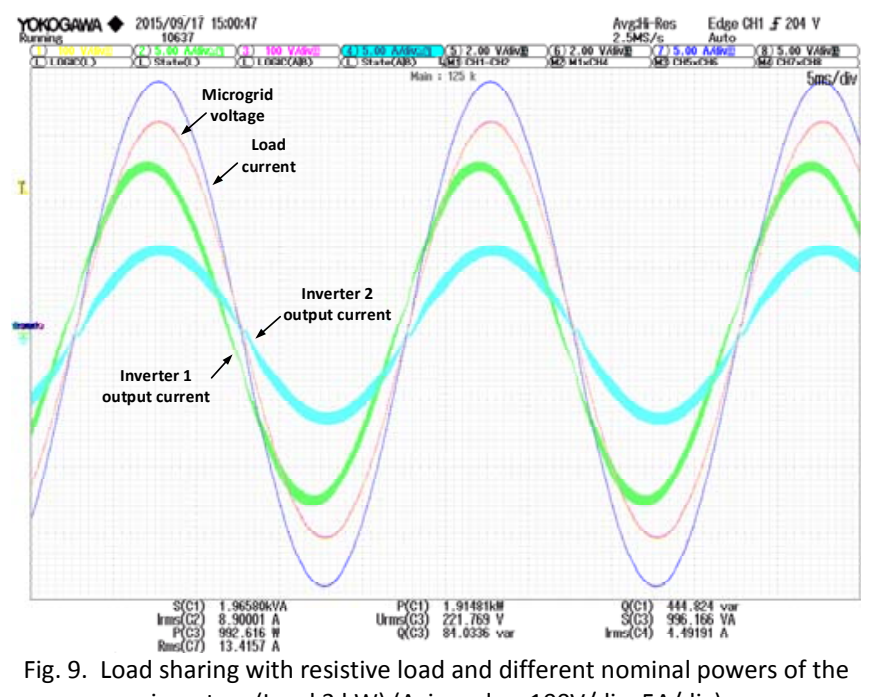

Fig. 9. Load sharing with resistive load and different nominal powers of the inverters (Load $3 \mathrm{~kW}$ ) (Axis scales: 100V/div; 5A/div)

A non-linear load of $1.55 \mathrm{kVA}(\mathrm{CF}=1.6, \mathrm{DPF}=1)$ has been also tested (figure 10.a). Inverter \#1 is loaded with $1.05 \mathrm{kVA}$ (52.5\% of its nominal output power), whereas inverter 2 is loaded with $0.55 \mathrm{kVA}$ ( $55 \%$ of its nominal output power), resulting that the power sharing remains in the expected values. Moreover, an RL load of $1.41 \mathrm{kVA} / \mathrm{PF}=0.71$ is also tested in the microgrid (figure 10.b), loading the inverters with the $45 \%$ and the $62.8 \%$ of the nominal apparent output power of each inverter.

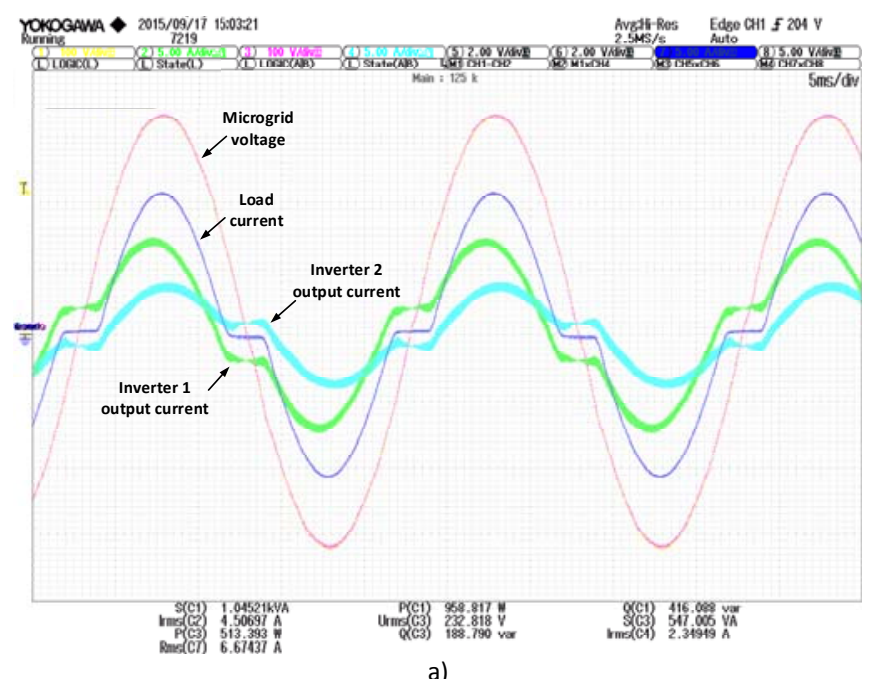

a)

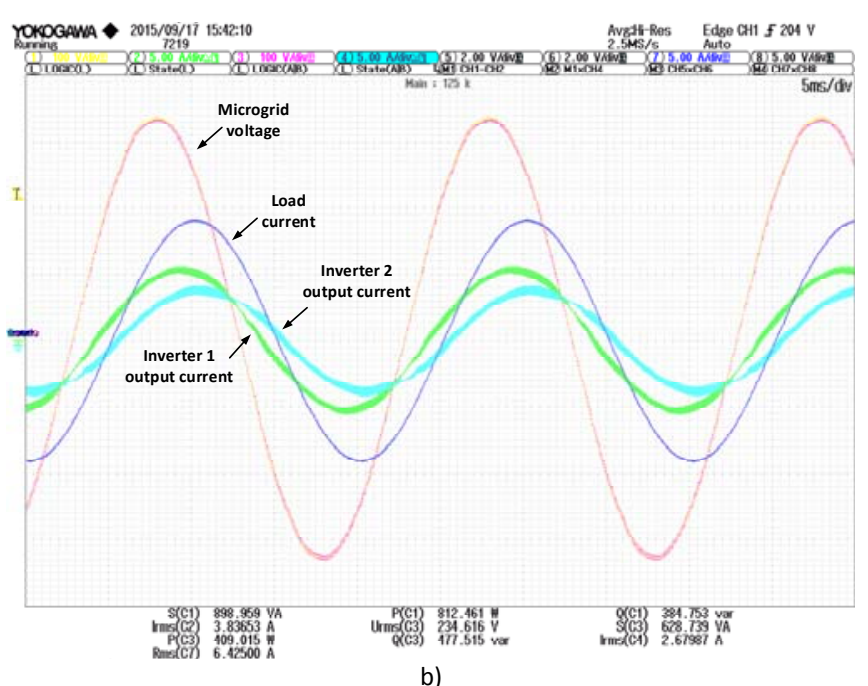

Fig. 10. Load sharing with different nominal powers of the inverters $A$ ) Nonlinear load $=1.55 \mathrm{kVA}, \mathrm{CF}=1.6, \mathrm{DPF}=1 \mathrm{~b}) \mathrm{RL}$ Load $=1.41 \mathrm{kVA} / \mathrm{PF}=0.71$ (Axis scales: $100 \mathrm{~V} /$ div; 5A/div)

In the following, a higher unbalance in the output nominal powers of the inverters is tested. The inverters are configured as shown in table IV. The output impedance of the inverter 1 is set to $0.5 \Omega$ (nominal power $5 \mathrm{kVA}$ ), while the output impedance of inverter 2 is adjusted to $5 \Omega$ (nominal power $1 \mathrm{kVA})$.

TABLE IV: GRID-FORMING CONVERTERS CONNECTED IN PARALLEL

\begin{tabular}{|l|c|c|}
\hline & Inverter 1 & Inverter 2 \\
\hline Nominal Output Voltage & \multicolumn{2}{|c|}{$230 \mathrm{~V} @ 50 \mathrm{~Hz}$} \\
\hline Nominal Output Power & $5 \mathrm{kVA}$ & $1 \mathrm{kVA}$ \\
\hline Output Virtual Impedance & $0.5 \Omega$ & $5 \Omega$ \\
\hline
\end{tabular}

Figure 11.a shows the load sharing achieved with a highly resistive load of $3 \mathrm{~kW}$. Inverter \#1 delivers $2.43 \mathrm{kVA}$ and \#inverter 2 delivers $0.59 \mathrm{kVA}$, being loaded with $48.6 \%$ and $58.7 \%$, respectively, of their maximum capacity. In figure $11 . \mathrm{b}$ it is tested an RL load with a poor power factor $(\mathrm{P}=1.41 \mathrm{~kW} /$ $\mathrm{PF}=0.71)$. Inverter $\# 1$ delivers $1.12 \mathrm{kVA}$ and inverter \#2 
delivers $0.5 \mathrm{kVA}$, corresponding to $22 \%$ and $50 \%$ of the nominal output power of each inverter. Under the extreme test conditions shown in table III, the load distribution is highly unbalanced. However, it is clearly shown that the voltage waveform is correctly generated; and the inverters on stream perform a stable behavior.
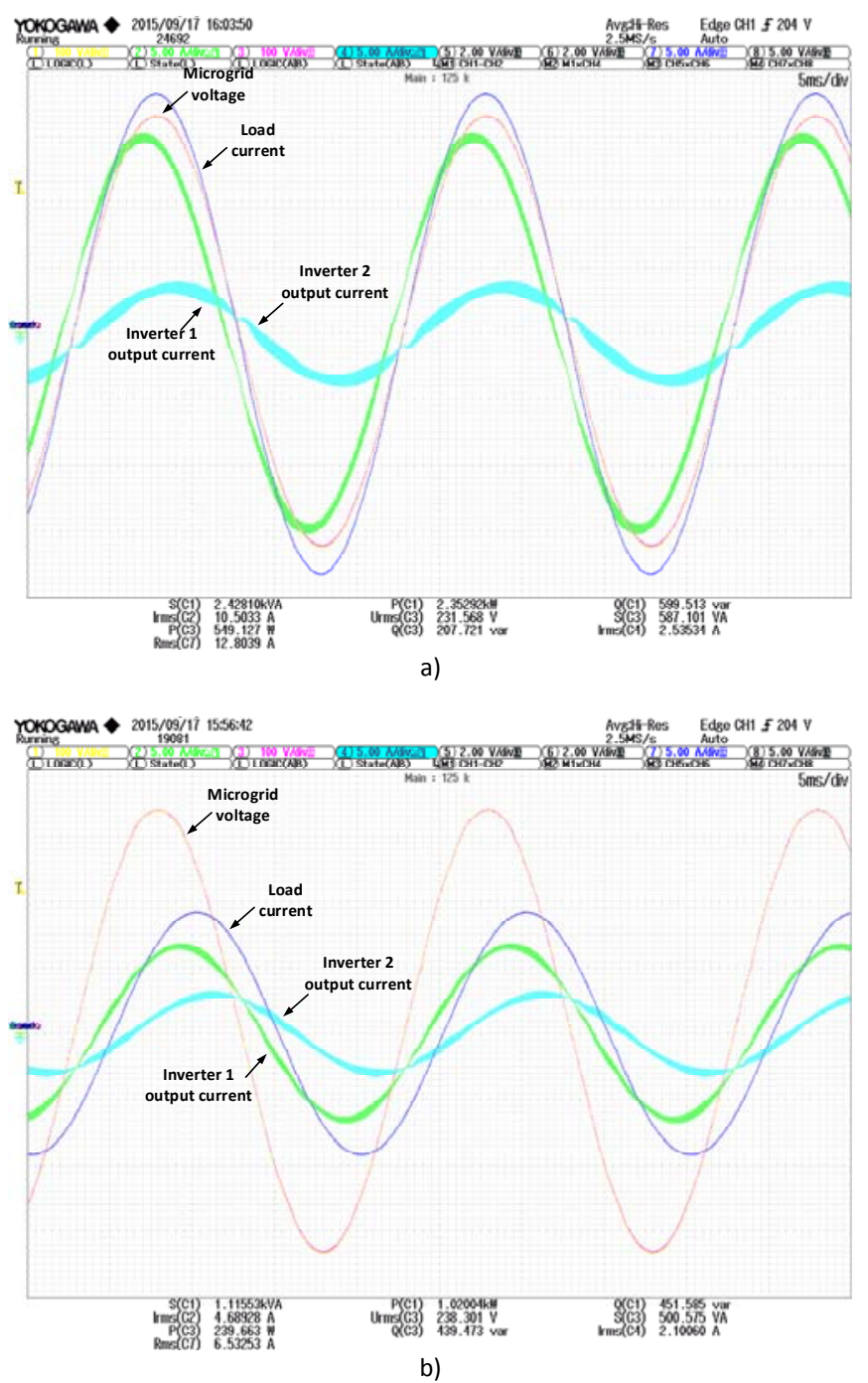

Fig. 11. Load sharing with very different nominal powers of the inverters. A) Resistive load $=3 \mathrm{~kW}$ b) RL load $=1.41 \mathrm{~kW} / \mathrm{PF}=0.71$ (Axis scales: $100 \mathrm{~V} / \mathrm{div}$; $5 \mathrm{~A} /$ div)

\section{CONCLUSIONS}

The FM modulation and the RS-485 transmission protocol over a low cost medium like twisted pair guarantees high noise immunity and a low time delay in the transmission of the synchronization signal among the grid-forming inverters in a microgrid. The laboratory tests demonstrate that the transmission medium is very robust to noise and that a small delay in the received signals is achieved in spite of a great distance among the inverters.

The output voltage of the grid-forming inverters can be adjusted in phase and frequency to that provided by the modulated signal. The modulation allows the parallel operation of several inverters with a similar behavior to that of a single voltage source whose nominal power is the sum of the powers of the individual inverters. The microgrid voltage can be correctly established, even using dispersed power converters in the microgrid.

Moreover, the use of different programmable output virtual impedances in each inverter achieves an acceptable load sharing among inverters. Experimental results have been obtained for different combinations of the nominal output powers of the inverters, confirming the correct sharing of the output power, even under poor power factor and non-linear loads. The higher power unbalance between inverters tested $(1 \mathrm{kVA}$ and $5 \mathrm{kVA})$ demonstrates that the synchronization technique presented in this paper is valid for a realistic microgrid scenario.

\section{REFERENCES}

[1] Wu, D.; Tang, F.; Dragicevic, T.; Vasquez, J.C.; Guerrero, J.M., "A Control Architecture to Coordinate Renewable Energy Sources and Energy Storage Systems in Islanded Microgrids," Smart Grid, IEEE Transactions on , no.99, pp.1,1.

[2] Micallef, A.; Apap, M.; Spiteri-Staines, C.; Guerrero, J.M., "SinglePhase Microgrid With Seamless Transition Capabilities Between Modes of Operation," in Smart Grid, IEEE Transactions on , vol.PP, no.99, pp.1-1.

[3] Peter Dondi, Deia Bayoumi, Christoph Haederli, Danny Julian, Marco Suter, "Network integration of distributed power generation", Journal of Power Sources, Volume 106, Issues 1-2, 1 April 2002, Pages 1-9.

[4] IEEE standard for interconnecting distributed resources with electric power systems. IEEE Std 1547-2003; 2003 p. 0-16.

[5] Ramon Zamora, Anurag K. Srivastava, "Controls for microgrids with storage: Review, challenges, and research needs", Renewable and Sustainable Energy Reviews, Volume 14, Issue 7, September 2010, Pages 2009-2018

[6] Huang Jiayi, Jiang Chuanwen, Xu Rong, "A review on distributed energy resources and MicroGrid", Renewable and Sustainable Energy Reviews, Volume 12, Issue 9, December 2008, Pages 2472-2483, ISSN 1364-0321

[7] Rocabert, J.; Luna, A.; Blaabjerg, F.; Rodríguez, P., "Control of Power Converters in AC Microgrids," Power Electronics, IEEE Transactions on vol.27, no.11, pp.4734,4749, Nov. 2012.

[8] Ciobotaru, M.; Teodorescu, R.; Blaabjerg, F., "A New Single-Phase PLL Structure Based on Second Order Generalized Integrator," in Power Electronics Specialists Conference, 2006. PESC '06. 37th IEEE , vol., no., pp.1-6, 18-22 June 2006

[9] Guerrero, J.M.; Matas, J.; Luis Garcia de Vicuna; Castilla, M.; Miret, J., "Decentralized Control for Parallel Operation of Distributed Generation Inverters Using Resistive Output Impedance," Industrial Electronics, IEEE Transactions on , vol.54, no.2, pp.994,1004, April 2007.

[10] Ashabani, M.; Moamed, Y.A.-.I.; Mirsalim, M.; Aghashabani, M., "Multivariable Droop Control of Synchronous Current Converters in Weak Grids/Microgrids With Decoupled dq-Axes Currents," Smart Grid, IEEE Transactions on , no.99, pp.1,1.

[11] H. Han, X. Hou, J. Yang, J. Wu, M. Su and J. M. Guerrero, "Review of Power Sharing Control Strategies for Islanding Operation of AC Microgrids," in IEEE Transactions on Smart Grid, vol. 7, no. 1, pp. 200215, Jan. 2016.

[12] C. Li; S. K. Chaudhary; M. Savaghebi; J. C. Vasquez; J. M. Guerrero, "Power Flow Analysis for Low-voltage AC and DC Microgrids Considering Droop Control and Virtual Impedance," in IEEE Transactions on Smart Grid, vol.PP, no.99, pp.1-1

[13] K. Yu, Q. Ai, S. Wang, J. Ni and T. Lv, "Analysis and Optimization of Droop Controller for Microgrid System Based on Small-Signal Dynamic Model," in IEEE Transactions on Smart Grid, vol. 7, no. 2, pp. 695-705, March 2016.

[14] X. Tang, X. Hu, N. Li, W. Deng and G. Zhang, "A Novel Frequency and Voltage Control Method for Islanded Microgrid Based on Multienergy 
Storages," in IEEE Transactions on Smart Grid, vol. 7, no. 1, pp. 410419, Jan. 2016

[15] A. Solanki, A. Nasiri, V. Bhavaraju, Y. L. Familiant and Q. Fu, "A New Framework for Microgrid Management: Virtual Droop Control," in IEEE Transactions on Smart Grid, vol. 7, no. 2, pp. 554-566, March 2016.

[16] Q. Li, F. Chen, M. Chen, J. M. Guerrero and D. Abbott, "Agent-Based Decentralized Control Method for Islanded Microgrids," in IEEE Transactions on Smart Grid, vol. 7, no. 2, pp. 637-649, March 2016.

[17] Changhee Cho; Jin-Hong Jeon; Jong-Yul Kim; Soonman Kwon; Kyongyop Park; Sungshin Kim, "Active Synchronizing Control of a Microgrid," Power Electronics, IEEE Transactions on , vol.26, no.12, pp.3707,3719, Dec. 2011.

[18] Qiang Fu; Nasiri, A.; Bhavaraju, V.; Solanki, A.; Abdallah, T.; Yu, D.C., "Transition Management of Microgrids With High Penetration of Renewable Energy," Smart Grid, IEEE Transactions on , vol.5, no.2, pp.539,549, March 2014.

[19] Junbo Sun; Da Xie; Yucheng Lou; Minxia Yang; Yu Zhang, "Blackstart control for EV's intelligent integrated station," Electricity Distribution (CICED), 2014 China International Conference on , vol., no., pp.1414,1419, 23-26 Sept. 2014.

[20] International Standard ISO/IEC 11801 Information Technology Generic Cabling for customer premises, Sept. 2002

[21] TMS320x2833x, 2823x Enhanced Capture (eCAP) Module. Reference Guide. Rev. A. June 2009. http://www.ti.com/lit/pdf/sprufg4

\section{BIOGRAPHIES}

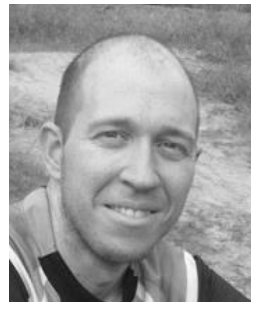

Iván Patrao was born in Valencia, Spain, in 1984. He received the Ingeniero Industrial (M.Sc) degree, in 2009 and the Dr. Ingeniería Electrónica Ph.D. degree in 2015, from the Universidad Politécnica de Valencia (UPV), Spain.

In 2008 he was with the R\&D department Siliken S.A., where he is involved in the design of electronic systems applied to photovoltaic applications. From 2009, he has been with the Electronics Engineering Department, UPV, where he was with the Industrial Electronic Systems Group (GSEI) http://gsei.upv.es. His main research fields are power converter modeling and control, converters for renewable energy, transformerless inverters and integration of distributed power sources in microgrids.

From 2014, Iván is working also in the engineering unit of La Florida University, Valencia, Spain, teaching in the electrical and electronical area.

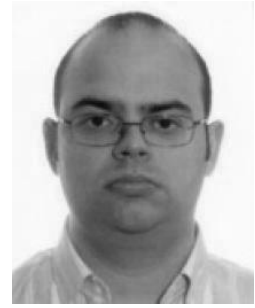

Raúl González-Medina was born in Valencia Spain, in 1978. He received the Ingeniero Industrial (M.Sc.) degree in 2005 and the Dr. en Ingeniería Electrónica Ph.D. degree in 2015, from the Universidad Politécnica de Valencia (UPV).

In 2005, he joined the Department of Electronics Engineering at the UPV, and got involved with the Industrial Electronic System Group (GSEI) where he is an R\&D engineer, focused on technology transfer to companies. His main research fields are power converters, modulation techniques, grid-connected inverters, and converter for renewable energy sources.

Since 2015, he is also teaching power electronics in the Department of Electronic Engineering at the UPV.

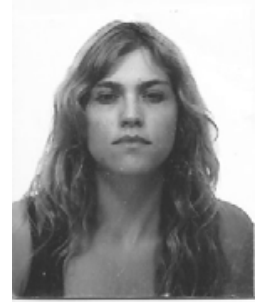

Silvia Marzal was born in Valencia, Spain, in 1984 $\mathrm{He}$ received the Ingeniero Telecomunicaciones (M.Sc) degree, in 2010 from the Universidad Politécnica de Valencia (UPV), Spain and she is currently working on her Ph.D. degree studies on Universidad Politécnica de Valencia (UPV), Spain.

In 2012 he was with the Institute Center for Transportation and Territory on Universidad Politécnica de Valencia (UPV), where she was involved in the design of communications systems applied to railway applications. From 2014,she has been with the Electronic Engineering Department, UPV, where he is with the Industrial Electronic Systems Group (GSEI), http://gsei.upv.es.Her main research fields are communications protocols and topologies design and development, to control and manage the distributed power sources in microgrids.

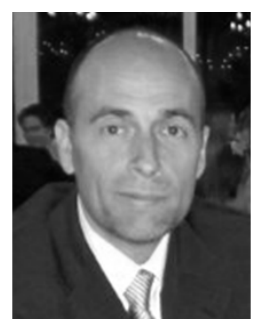

Gabriel Garcerá (IEEE Student member 1997 Associate member 1999, Member 2004) received the Ingeniero de Telecomunicación (M.Sc.) and Dr Ingeniero de Telecomunicación (Ph.D.) degrees from the Polytechnic University of Valencia in 1993 and 1998 , respectively

From 1993 to 1995 he was with the R\&D Department of the company GH ELIN International (currently GH Group), involved in the design of high current switch-mode power supplies for particle accelerator superconducting magnets at CERN. By the end of 1995 he joined the Electronics Engineering Department of the Polytechnic University of Valencia, where he is currently a Full Professor and the Coordinator of the Industrial Electronic Systems Group (GSEI), http://gsei.upv.es. His main research fields are in power converter modeling and control, power factor correction circuits, uninterruptible power supplies, grid-connected inverters, power converters for renewable energy sources and distributed generation microgrids. He is coauthor of more than 100 papers about electronic power conversion published in international journals and conferences. Besides, he has advised 13 Ph.D. Thesis about Power Electronics. He was a co-recipient of the best paper award published in 2012 in the IEEE Transactions on Industrial Electronics.

Dr. Garcerá is a member of the IEEE. Since 2004, he is an Associate Editor of the IEEE Transactions on Industrial Electronics.

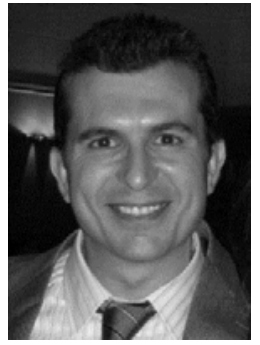

Emilio Figueres (S'98-A'00-SM'10) received the M.Sc. degree from the Ecole Nationale Supérieure d'Electrotechnique, d'Electronique, d'Informatique et d'Hydraulique de Toulouse, Toulouse, France, in 1995, and the Dr. Ingeniero Industrial (Ph.D.) degree from the Universidad Politécnica de Valencia (UPV), Valencia, Spain, in 2001. Since 1996, he has been with the Electronics Engineering Department, UPV, where he is currently a Full Professor and the Head of the Department. His main research interests include modeling and control of power converters, power processing of renewable energy sources, and grid connected converters for distributed power generation and improvement of power quality. In these fields, he has coauthored more than 100 papers published in JCR indexed journals and conferences. He also holds 3 patents and was a co-recipient of the best paper award published in 2012 in the IEEE Transactions on Industrial Electronics. 